\title{
Spread Films of Human Serum Albumin at the Air-Water Interface: Optimization, Morphology, and Durability
}

\author{
Richard A. Campbell ${ }^{*}$, Joo Chuan Ang ${ }^{b}$, \\ Federica Sebastiani ${ }^{a, c}$, Andrea Tummino ${ }^{a, d}$ and John W. White ${ }^{b^{*}}$
}

${ }^{a}$ Institut Laue-Langevin, 71 avenue des Martyrs, CS 20156, 38042 Grenoble, France.

${ }^{\mathrm{b}}$ Research School of Chemistry, Australian National University, Canberra, ACT 0200, Australia.

${ }^{c}$ Department of Chemistry, University of Reading, PO Box 224, Reading RG6 6AD, UK.

${ }^{\mathrm{d}}$ Institute of Chemistry, Eötvös Loránd University, Budapest 112, H-1518 Hungary.

\section{Supporting Information}

\section{Part 1. Neutron reflectivity and scattering length density profiles}

Figure SI1 shows examples of the neutron reflectivity and real space scattering length density profiles used to calculate the surface excess values in figure 1 of the main text.
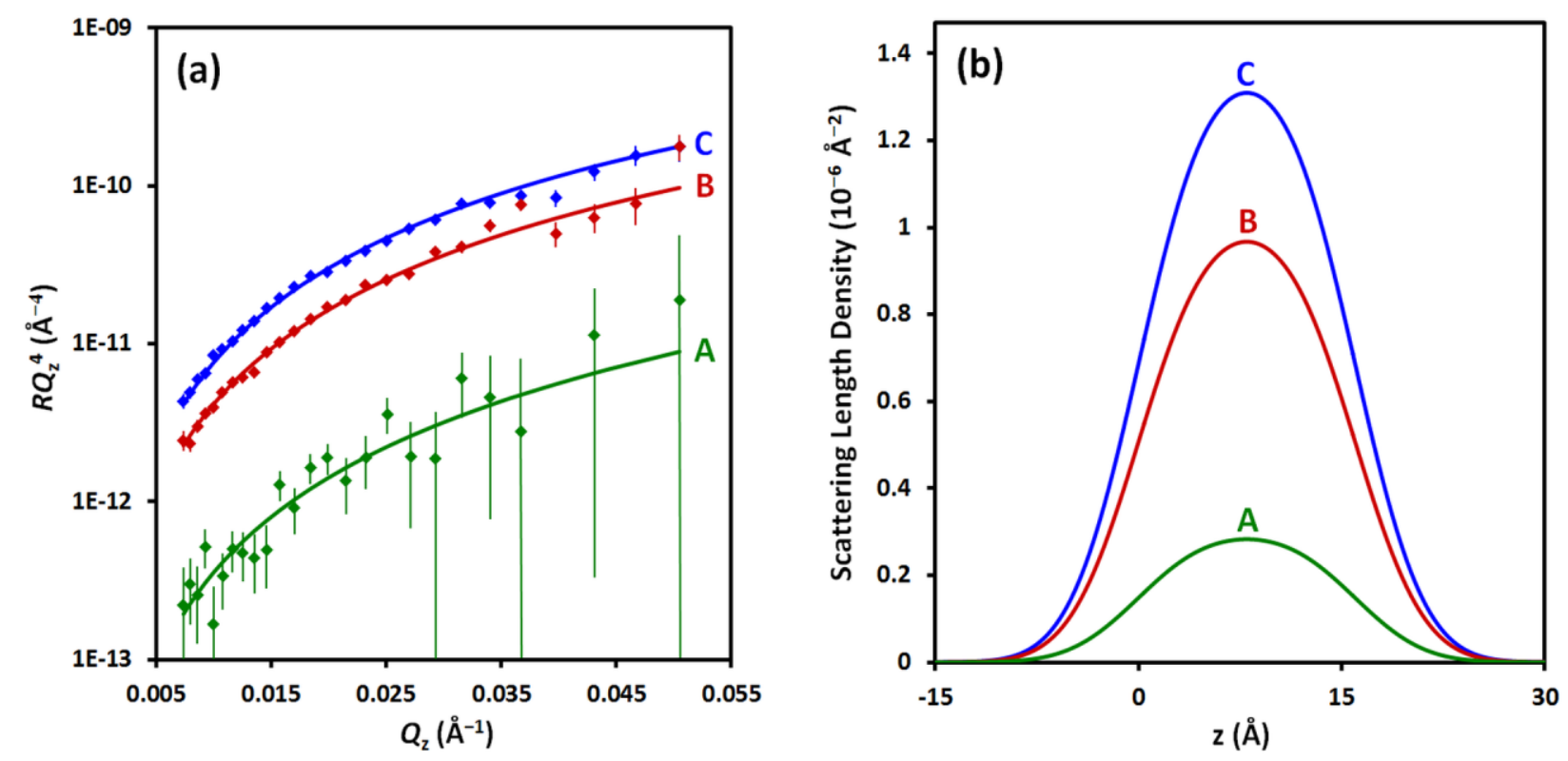

Figure SI1. (a) Neutron reflectivity profiles and (b) real space scattering length density profiles for adsorbed layers of DF-HSA from aspirated, premixed solutions in PBS with bulk concentrations of (A; green) 0.001 , (B; red) 0.02 and $\left(\mathrm{C}\right.$; blue) $0.5 \mathrm{mg} / \mathrm{cm}^{3}$. The samples were aspirated, the surface age was $\sim 30 \mathrm{~min}$, and the data acquisition time was 5 min. 


\section{Part 2. Ellipsometry data analysis}

In ellipsometry measurements, we define the effect of the surface layer on the measured phase shift as $\Delta_{\text {surf }}=\Delta-\Delta_{0}$, where $\Delta$ is the measured parameter for a protein solution and $\Delta_{0}$ is the measured reference value for pure water. This approach neglects changes in the scattering by thermal capillary waves for different samples as a result of the minor influence of the changing surface tension $^{\text {SII }}$, i.e., the roughness contribution to $\Delta_{\text {surf }}$ is minimized as a result of subtraction of the reference $\Delta_{0}$ measurement.

In the thin film limit, $\Delta_{\text {surf }}$ is linearly proportional to the ellipsometric thickness $\eta$, S12

$\Delta_{\text {surf }}=\frac{g(\theta)}{\lambda} \cdot \eta$

where $g(\theta)$ is a function that depends only on bulk properties and on the angle of incidence, ${ }^{\text {S12 }}$

$g(\theta)=\frac{4 \pi \cdot n_{\mathrm{air}} \cdot n_{\mathrm{water}}{ }^{2} \cdot \cos \theta \cdot \sin ^{2} \theta}{\left(n_{\mathrm{water}}{ }^{2}-n_{\mathrm{air}}{ }^{2}\right) \cdot\left(n_{\mathrm{water}}{ }^{2}+n_{\mathrm{air}}{ }^{2}\right) \cdot \cos ^{2} \theta-n_{\mathrm{air}}{ }^{2}}$

The term $\eta$ can be written in terms of the refractive index profile across the interface, ${ }^{\mathrm{SI} 3}$

$\eta=\int \frac{\left(n(z)^{2}-n_{\text {air }}^{2}\right)\left(n(z)^{2}-n_{\text {water }}^{2}\right)}{n(z)^{2}}$

where $n(z)$ is the refractive index profile in the direction perpendicular to the interface, $n_{\mathrm{air}}=1.000$ is the refractive index of air and $n_{\text {water }}=1.332$ is the refractive index of water. For a single uniform isotropic layer separated by sharp stratified interfaces, eqs SI1 and SI3 may be reduced to an expression for $\Delta_{\text {surf }}$ in terms of $\tau$ the film thickness (or $\rho$ the film density)

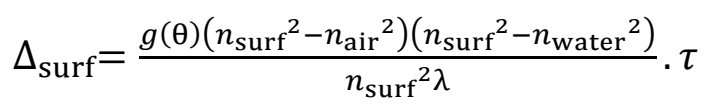

where $n_{\text {surf }}$ is the refractive index of the surface layer of protein and solvent. Next, de Feijter's equation can be used to derive a relation between the surface excess $\Gamma$ and $\Delta_{\text {surf, }}$, ${ }^{4}$

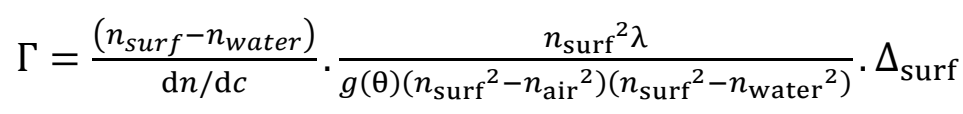

where $\mathrm{d} n / \mathrm{d} c$ is the refractive index increment for DF-HSA solutions.

One has to choose whether to model changes in the surface coverage of DF-HSA as changes to the layer thickness at constant density ('oil-like' behavior) or changes to the layer density or coverage at constant thickness ('particle-like' behavior). In the former case of constant layer density there is no solvent in the surface layer, so $n_{\text {surf }}$ is a constant, hence the relation between the $\Gamma$ and $\Delta_{\text {surf }}$ is linear. To derive the relation, the main input parameters into the function are the refractive index increment $\left(0.186 \mathrm{~cm}^{3} / \mathrm{g}\right)^{\mathrm{SIS}}$ and the density of the protein, which was calculated as $1.292 \mathrm{~g} / \mathrm{cm}^{3}$ from 
the molecular weight of $66437 \mathrm{~g} / \mathrm{mol}^{\mathrm{SI} 6}$ and the molecular volume of $85433 \AA^{3}$, itself calculated from the average values resulting from published crystallographic data. ${ }^{\text {SI7-SI9 }}$ These give the relation $\Gamma=0.495 . \Delta_{\text {surf }}$

where the units of $\Gamma$ are $\mathrm{mg} / \mathrm{m}^{2}$ and the units of $\Delta_{\text {surf }}$ are degrees. In the latter case of constant layer thickness, $n_{\text {surf }}$ is a variable related to the density or coverage of protein in the layer, hence the relation between the surface excess and optical phase shift is now a quadratic expression. An empirical fit to simulated data for the same parameters listed above, using a layer thickness of $16 \AA$ (consistent with the structure of the kinetically-trapped film on a dilute subphase determined from figure 3 in the main text), gives the relation

$\Gamma=0.632 . \Delta_{\text {surf }}-0.0440 . \Delta_{\text {surf }}^{2}$

Note that the surface excess values calculated using eq. SI7 are affected by only a few percent by the choice of layer thickness in the thin film limit. A comparison of the effects of using eqs SI6 and SI7 is shown in figure SI2.

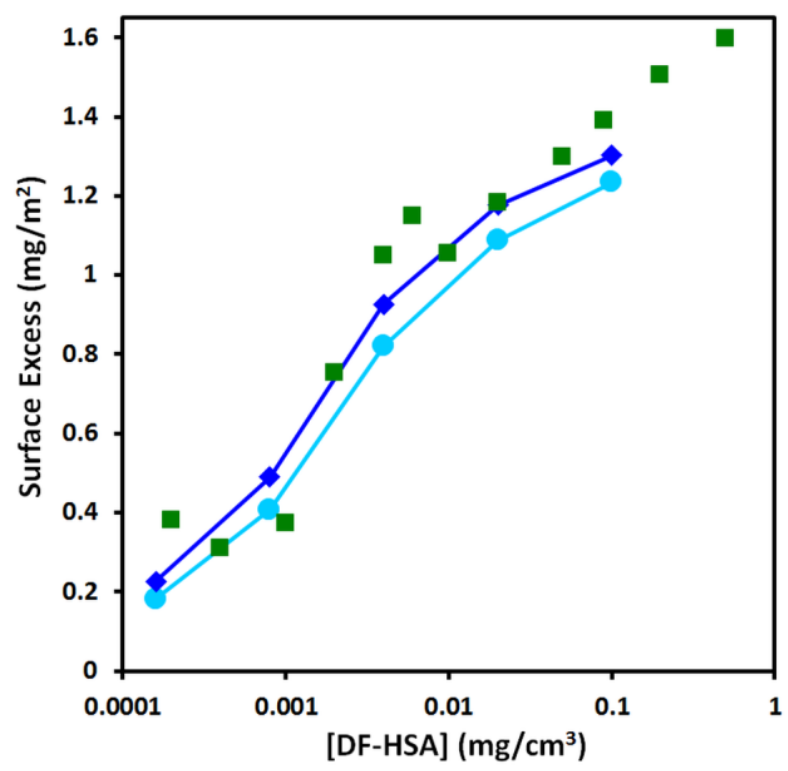

Figure SI2. A comparison of surface excesses of adsorbed layers of DF-HSA from aspirated, premixed solutions in PBS calculated from ellipsometry data using eqs SI6 (pale blue circles; oil-like model) and SI7 (blue diamonds; particle-like model). The values from NR are shown for reference (green squares). Lines joining the data are a guide to the eye.

The surface excess values calculated using the model of changing layer density or coverage rather than thickness agree slightly better with the values measured using NR. Further, the structure of the kinetically-trapped film of DF-HSA on a dilute subphase from XRR (figure 3; main text) is a single layer of $16 \AA$, and one would not expect the thickness to be significantly less at lower surface 
coverage due to conservation of the primary protein structure. Therefore eq. SI7 rather than eq. SI6 was chosen to carry out the surface excess calculations in the main text.

\section{Part 3. X-ray reflectivity profiles}

Figure SI3 shows the X-ray reflectivity profiles used to generate the electronic density profiles in figure 3 of the main text.

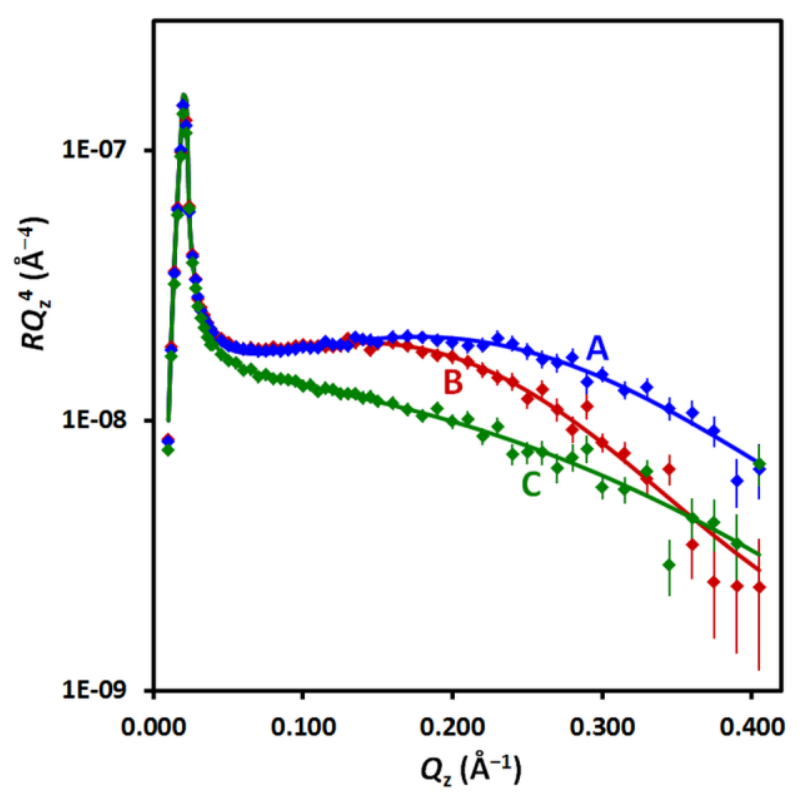

Figure SI3. X-ray reflectivity profiles for a spread film of DF-HSA using 5 droplets of 1 $\mathrm{mg} / \mathrm{cm}^{3} \mathrm{DF}-\mathrm{HSA}$ as the dispensing solution on PBS in a flow trough both (A; blue) before and (B; red) after exchanging the subphase; data from the water is shown as a reference (C; green).

\section{Part 4. Analysis of the protein adsorption kinetics}

The adsorption kinetics of DF-HSA at the air-water interface, characterized using ellipsometry, are shown in figure 6 of the main text. From the work of Ramsden, ${ }^{\text {SI10 }}$ the initial adsorption of protein to a clean interface is related to $D^{2 / 3}$ (where $D$ is the diffusion coefficient), the bulk concentration $c$, and the square root of the time from surface creation $t^{1 / 2}$. Each curve in figure 6 has a roughly linear component at short times before the slopes decrease as the interface starts to become jammed. The first three gradients of these initial linear regions are linear with a positive slope with respect to $c$, as expected from Ramsden; ${ }^{\mathrm{SI} 10}$ see the left panel of figure SI4. In contrast, the last three gradients are linear with a negative slope with respect to $c$, showing that the jamming limit is reached even in the initial stages of adsorption at higher bulk protein concentrations; see the right panel of figure SI4. HSA in solution at $\mathrm{pH} 7$ carries a net positive charge of $\sim 40$ (the isoelectric point is 8.2 ). As the 
molecular density at the surface increases, the consequent intermolecular electrostatic interaction may be the principle cause of the jamming effect observed.

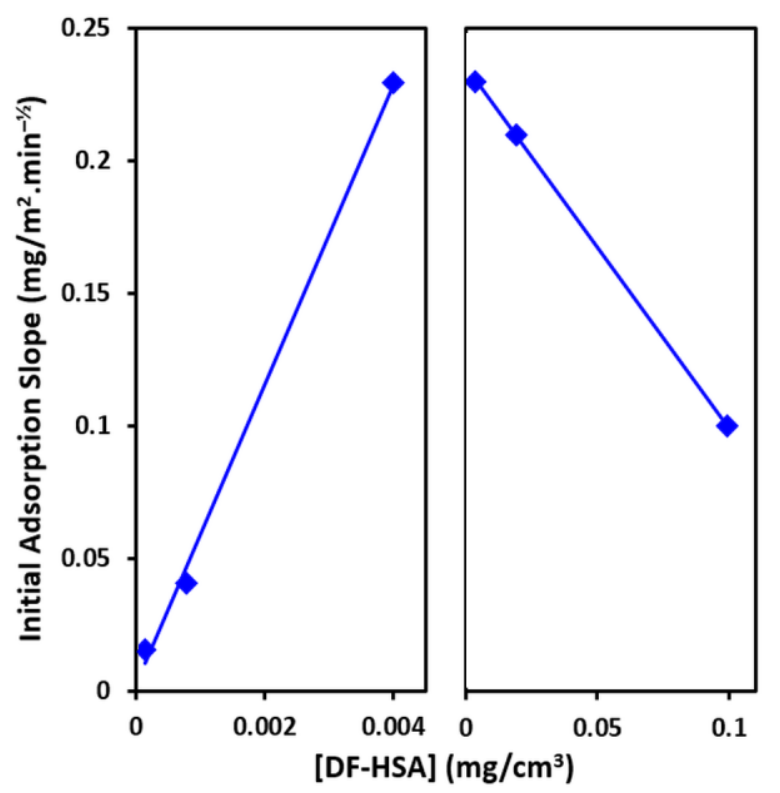

Figure SI4. Initial adsorption slope of the surface excess vs. $t^{1 / 2}$ data from figure 6 of the main text with respect to the bulk DF-HSA concentration. The data fall into two linear regimes: the first (left) where protein fills in gaps at the air-water interface at the diffusion-controlled rate, and the second (right) where protein experiences jamming even at short adsorption times.

\section{Part 5. Additional compression/expansion isotherms}

To examine effects of changing the rheology on the annealing process, a surface pressure/area isotherm experiment analogous to that described in the main text was carried out at half the cycling rate. Figure SI5 shows (A) the surface pressure and (B) the ellipsometry data measured.

The adsorbed layer reaches higher surface pressures than those achieved in the experiment at the higher cycling rate. This difference supports our interpretation that the increasing maximum surface pressures reached can be attributed to the adsorption of protein from the bulk to form a substantial surface layer. The minimal hysteresis again in this experiment, even when the maximum surface pressure is almost the same as that reached in the spread films, indicates that the morphologies of the adsorbed layer and spread films are different. This interpretation is supported by the features observed in the BAM images reported in the main text.

Both the surface pressure and ellipsometry data from the spread film are similar to those recorded at the higher cycling rate. The surface pressure limit cycle is approached in earlier cycles although it should be noted that each one took longer. These small changes suggest that there is at least potential to tune rheological aspects to optimize annealing of the morphology of the spread films. 

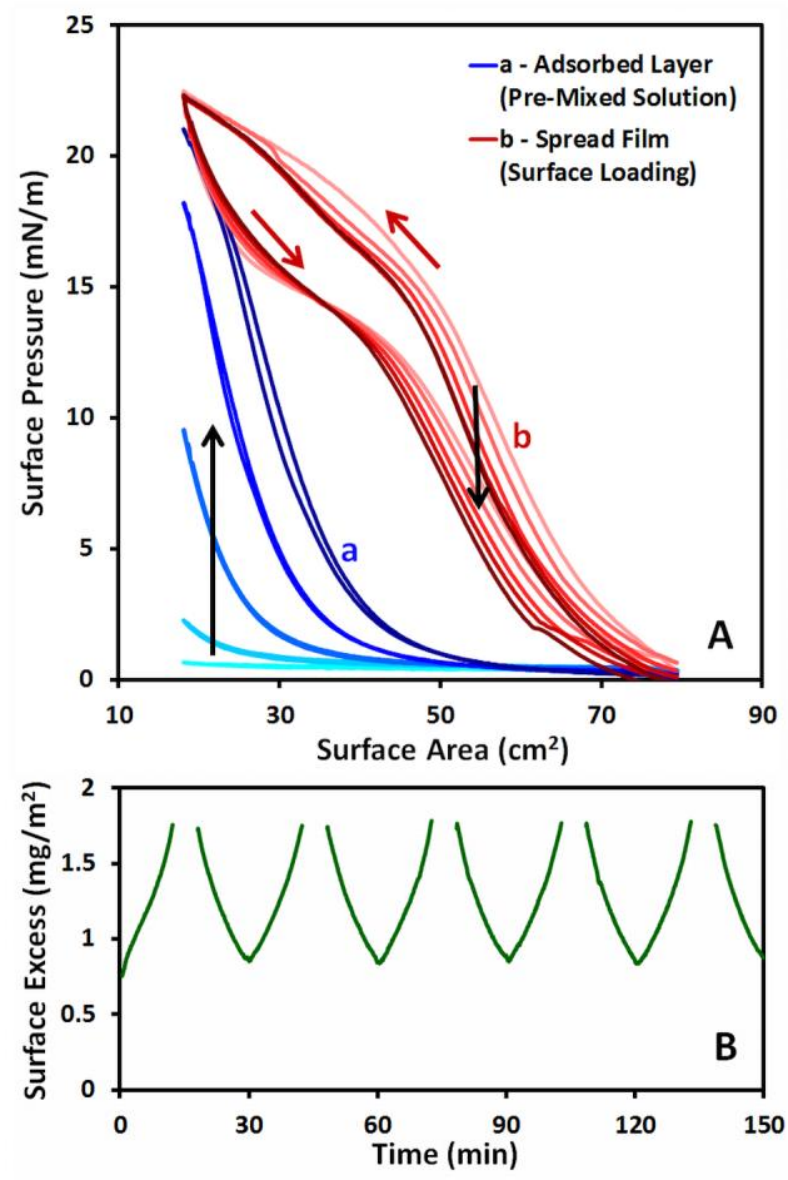

Figure SI5. (A) Surface pressure and (B) ellipsometry data analogous to those presented in figure 7 of the main text but at half the compression/expansion rate, i.e., a cycle time of 29 min using a constant compression and expansion speed on each barrier of $4.1 \mathrm{~mm} / \mathrm{min}$.

\section{References}

SI1. Meunier, J. Light Scattering by Liquid Surfaces and Complementary Techniques; Marcel Dekker: New York, 1992.

SI2. Motschmann, H.; Teppner, R. Ellipsometry in Interface Science; Elsevier: New York, 2001.

SI3. Drude, P. Ueber die reflexion und brechung ebener lichtwellen beim durchgang durch eine mit oberflächenschichten behaftete planparallele platte. Annalen der Physik 1891, 43, 126-157.

SI4. Defeijter, J. A.; Benjamins, J.; Veer, F. A. Ellipsometry as a tool to study the adsorption behavior of synthetic and biopolymers at the air-water interface. Biopolymers 1978, 17, 17591772 .

SI5. Wen, J.; Arakawa, T.; Philo, J. S. Size-exclusion chromatography with on-line light-scattering, absorbance, and refractive index detectors for studying proteins and their interactions. Analytical Biochemistry 1996, 240, 155-166. 
SI6. Meloun, B.; Morávek, L.; Kostka, V. Complete amino-acid sequence of human-serum albumin. FEBS Letters, 1975, 58, 134-137.

SI7. He, X. M.; Carter, D. C. Atomic structure and chemistry of human serum albumin. Nature 1992, 358, 209-215.

SI8. Curry, S.; Mandelkow, H.; Brick, P.; Franks, N. Crystal structure of human serum albumin complexed with fatty acid reveals an asymmetric distribution of binding sites. Nat. Struct. Biol. 1998, 5, 827-835.

SI9. Sugio, S.; Kashima, A.; Mokizuki, S.; Noda, M.; Kobayashi, K. Crystal structure of human serum albumin at 2.5 Å resolution. Protein Eng. 1999, 12, 439-446.

SI10. Ramsden, J. J. Concentration scaling of protein deposition kinetics. Phys. Rev. Lett. 1993, 71, 295-298. 